\title{
Anonymous Fair Auction on Blockchain
}

\author{
Gaurav Sharma \\ Cybersecurity Research Center \\ Université Libre de Bruxelles \\ Brussels, Belgium \\ gaurav.sharma@ulb.be
}

\author{
Denis Verstraeten \\ Cybersecurity Research Center \\ Université Libre de Bruxelles \\ Brussels, Belgium \\ denis.verstraeten@ulb.be
}

\author{
Vishal Saraswat \\ Engineering Product Security Solutions \\ Robert Bosch Engineering and Business Solutions \\ Bangalore, India \\ vishal.saraswat@gmail.com
}

\author{
Olivier Markowitch \\ Cybersecurity Research Center \\ Université Libre de Bruxelles \\ Brussels, Belgium \\ olivier.markowitch@ulb.be
}

\begin{abstract}
Online auction systems allow to achieve an optimal trading of digital goods and services in a competitive market. Existing blockchain-based auctions allow the bidders to take part in the process while maintaining the confidentiality of their bids in a decentralized, transparent, secure and auditable way. However, in a competitive market, parties would prefer not to disclose their interests to competitors, and to remain anonymous during auctions. In this paper, we firstly analyze the specific requirements for blockchain-based anonymous fair auctions. We propose a formal model tailored to study auction systems facilitating anonymity, and we put forward a generic protocol relying on existing cryptographic primitives, such as designated verifier ring signature, to achieve bid confidentiality and bidder anonymity. Finally, we prove it to be secure against the security model we defined.

Index Terms-blockchain, auction, anonymity, designated identity verifier ring signature
\end{abstract}

\section{INTRODUCTION}

A distributed ledger of transactions providing record consistency and immutability is well admired in the domain of cryptocurrencies. The decentralization inherent to blockchains currently offers several properties including transparency and integrity. However, some applications require other security features, such as privacy. This last property is still unconventional in blockchain systems and is not straightforward to achieve, requiring the use of cryptographic techniques.

A well designed auction system with a thorough understanding of bidding behavior can allocate resources in accordance with expected outcome. Different environments and settings define particular auction types. One of the most popular is the sealed-bid auction, where each bidder hands over a sealed envelope containing their secret bid to the auctioneer. In this paper, we focus on first-price sealed-bid auction, where the highest bidder is the winner and has to pay the highest bid.

The auction process has to be managed by an auctioneer, who is the designated party responsible for dispatching the auction resources. Ideally, she is trusted and should not collude with any of the bidders to outcome a biased decision. However, reducing the trust on the auctioneer is an innovative feature in line with the philosophy of blockchains, this is why our contribution aims to expand the abilities of such blockchainbased auction systems.

\section{A. Related Work}

A large number of the protocols presented in the literature are based on the Ethereum blockchain, which is fully open and lacks privacy by default, but features a rich programmable environment relying on the smart contract paradigm. Kosba et al. proposed the addition of privacy to Ethereum in [7] by using privacy preserving smart contracts, to ensure transactional privacy. Their framework is versatile and can accommodate auctions systems.

In [2], the Strain protocol allows to build blockchain-based sealed-bid auctions preserving bids privacy against malicious parties. A public bulletin board is used to publish the winning bid which is determined by comparing them by pairs. Zero-Knowledge Proofs (ZKPs) are used to ensure that the participants used their original bid and that the auctioneer declared the winner without manipulation. Malicious bidders are punished by revealing their bid.

Galal and Youssef presented a smart contract for verifiable first-price sealed-bid auction in [6]. Bidders submit their bids to a smart contract using Pedersen commitment, which are secretly revealed to the auctioneer via a Public Key Encryption (PKE) scheme. After declaring the winner, for each losing bid, the auctioneer has to engage into a set of interactive commitchallenge-verify protocols and therefore, the complexity of interaction depends on the number of bidders. Later, they improved this protocol in [5] and presented a smart contract with succinctly verifiable ZKP which enables single proof verification for the whole auction process.

We observe that the existing literature focuses on bids confidentiality. To achieve bidder anonymity, the design of a new protocol needs to be introduced. 


\section{B. Auction in a Fair Market}

We assume a situation where all the bidders want to participate to an auction and to obtain their goods at a competitive price. In such an environment, they do not even want to disclose their interest, or the fact that they won the auction. This means that the identity of the bidders as well as the value of their bid should remain secret, and that the overall auction process should be publicly verifiable.

\section{Our Contribution}

We introduce a new protocol allowing to run an anonymous auction. Our construction presents the advantage of being generic and of using only existing cryptographic building blocks. Confidentiality and anonymity properties are achieved using Designated Verifier Ring Signature (DVRS), commitment and PKE. Precisely, our sealed-bid auction protocol enables the bidders not to disclose their bids (confidentiality) and their identity (anonymity); to put minimal trust on the auctioneer, as she is trusted to assist the process but is unable to affect the outcome; to prevent the bidders from modifying their bids during the auction (bid binding); and to be publicly verifiable, which is required in the event of a dispute.

\section{Preliminaries}

In this section, we recall some standard definitions of commitment scheme, PKE, Ring Signature (RS) and ZKP. In what follows, standard security definitions are assumed for the security properties of the schemes.

\section{A. Commitment Scheme}

A commitment scheme is 2-tuple of PPT algorithms Comm $=($ Commit, Open $)$ which needs to be correct, hiding and binding and which are described as follows:

Commitment: $(c, d) \leftarrow \operatorname{Commit}(m)$, where $(c, d)$ is the commitment pair and $m$ is a message.

Opening: $m \leftarrow$ Open $(c, d)$, where $(c, d)$ is a valid pair.

\section{B. Ring Signature}

Ring Signature was introduced by Rivest et al. in 2001 [8] and allows a member of a ring of users to anonymously sign a message. A ring is a list of possible signers, with each of them being associated with a public key $\mathrm{pk}_{i}$, and the corresponding secret key $\mathbf{s k}_{i}$ (for the $i^{\text {th }}$ user). When a verifier checks the signature, she can only conclude that a member of the ring endorsed it, without being able to determine the identity of the signer. A RS scheme should be correct, unforgeable and anonymous, and consists of a 3-tuple of PPT algorithms RS = (RGen, RSig, RVer) defined as follows:

Key Generation: $\left(\mathrm{pk}, \mathrm{sk}_{s}, s\right) \leftarrow \mathrm{RGen}\left(1^{k}\right)$, where $k$ denotes the security parameter, $\mathrm{pk}=\left[\mathrm{pk}_{1}, \ldots, \mathrm{pk}_{n}\right]$ is a list of $n$ public keys, which includes the public key of the signer and $n-1$ decoy public keys, $\mathbf{s k}_{s}$ is the secret key of the signer and $s$ is the index of the signer's public key $\mathrm{pk}_{s}$ in pk.

Signature: $\sigma \leftarrow \operatorname{RSig}\left(\mathrm{pk}_{\mathrm{sk}}, m\right)$, where $\sigma$ is the signature, and $m$ the message to be signed.
Verification: $b \leftarrow \operatorname{RVer}(\mathrm{pk}, m, \sigma)$, where outcome $b \in\{0,1\}$ indicates the validity of the signature.

\section{Public Key Encryption}

A PKE scheme consists of a 3-tuple of PPT algorithms PKE = (PKEGen, Enc, Dec) which should be correct and ciphertext indistinguishable and which are described as shown below:

Key Generation: (sk, pk) $\leftarrow \operatorname{PKEGen}\left(1^{k}\right)$, where $k$ is the security parameter, and pk and sk are the public and secret key, respectively.

Encryption: $c \leftarrow \operatorname{Enc}(\mathrm{pk}, m)$, where $m$ is the message and $c$ the ciphertext.

Decryption: $m \leftarrow \operatorname{Dec}($ sk, $c)$.

\section{Zero-Knowledge Proof}

A ZKP system is a 3-tuple of algorithms ZKP = (ZKGen, ZKProve, ZKVer) which should be perfectly complete, succint, computationally sound and computationally ZeroKnowledge. They are defined as follows:

Key Generation: CRS $\leftarrow \operatorname{ZKGen}\left(1^{k}, \mathcal{L}\right)$, where CRS is the common reference string, $k$ the security parameter, and $\mathcal{L}$ the language description.

Proof Generation: $\pi \leftarrow$ ZKProve $(\mathrm{CRS}, s, w)$, with $s$ the statement whose membership to $\mathcal{L}$ has to be proved, $w$ the corresponding witness, and $\pi$ the proof.

Proof Verification: $b \leftarrow \operatorname{ZKVer}(\mathrm{CRS}, \pi, s)$, with $b \in\{0,1\}$ the single bit indicating whether the verification succeeded.

\section{Formal Model ANd Security Definitions}

In this section, the mathematical definition of our Anonymous Fair Auction protocol are introduced, as well as the security properties which it has to fulfill. An Anonymous Bidding System (ABS) is a 4-tuple of PPT algorithms ABS = (KeyGen, Bid, BidOp, IdOp) defined as follows:

Key Generation: $\left(\mathrm{pk}, \mathrm{sk}_{s}, s, v\right) \leftarrow \operatorname{KeyGen}\left(1^{k}\right)$, with $k$ the security parameter, $\mathrm{pk}=\left[\mathrm{pk}_{1}, \ldots, \mathrm{pk}_{n}\right]$ the ring members' public key, sk ${ }_{s}$ the signer's private key, $s$ and $v$ the indices of the signer's and verifier's public key in pk, respectively.

Bid submission: $\left(c, \mathrm{sig}, \tau_{1}, \tau_{2}\right) \leftarrow \operatorname{Bid}\left(\mathrm{pk}, \mathrm{sk}_{s}, \mathrm{pk}_{v}, x\right)$, with $x$ the bid value, $\mathrm{pk}_{v}$ the verifier's public key, $c$ the commitment to the bid, sig the signature of the bid, $\tau_{1}$ the bid opening token and $\tau_{2}$ the identity opening token.

Bid opening: $b \leftarrow \operatorname{BidOp}\left(\mathrm{pk}, \mathrm{sk}_{v}, c, \mathrm{sig}, \tau_{1}\right)$, with $\mathrm{sk}_{v}$ the verifier's private key and $b \in\{0,1\}$ a single bit indicating whether the bid opening succeeded.

Identity opening: $b \leftarrow \operatorname{IdOp}\left(\mathrm{sk}_{v}\right.$, sig, $\left.\tau_{2}\right)$, with $b \in\{0,1\}$ a single bit indicating whether the identity opening succeeded.

Our ABS has the following security properties:

Correctness: It is correct if for any valid bid $x$, any $\left(\mathrm{pk}, \mathrm{sk}_{s}, s, v\right) \leftarrow \operatorname{KeyGen}\left(1^{k}\right)$, and $\left(c, \operatorname{sig}, \tau_{1}, \tau_{2}\right) \leftarrow$ $\operatorname{Bid}\left(\mathrm{pk}, \mathrm{sk}_{s}, \mathrm{pk}_{v}, x\right)$, the following two properties hold:

$$
\begin{aligned}
& \operatorname{Pr}\left[\operatorname{BidOp}\left(\mathrm{pk}_{,} \mathrm{sk}_{v}, c, \mathrm{sig}, \tau_{1}\right)=1\right]=1 ; \\
& \operatorname{Pr}\left[\operatorname{IdOp}\left(\mathrm{sk}_{v}, \mathrm{sig}, \tau_{2}\right)=1\right]=1 .
\end{aligned}
$$


Unforgeability: It satisfies unforgeability if for any PPT adversary $\mathcal{A}$,

$$
\operatorname{Pr}\left[\begin{array}{l}
\left(\mathrm{pk}, \mathrm{sk}_{s}, s, v\right) \leftarrow \operatorname{KeyGen}\left(1^{k}\right), \\
\left(x, c, \mathrm{sig}, \tau_{1}\right) \leftarrow \mathcal{A}^{\operatorname{Bid}\left(\mathrm{pk}, \mathrm{sk}_{s}, \mathrm{pk}_{v}, \cdot\right)(\mathrm{pk}),} \mid b=1 \\
b \leftarrow \operatorname{BidOp}\left(\mathrm{pk}, \mathrm{sk}_{v}, c, \mathrm{sig}, \tau_{1}\right)
\end{array}\right]
$$

is negl $(k)$ where the adversary $\mathcal{A}$ is not allowed to query the bid submission oracle $\operatorname{Bid}\left(\mathrm{pk}, \mathrm{sk}_{s}, \mathrm{pk}_{v}, \cdot\right)$ with bid $x$. Our ABS satisfies strong unforgeability if it satisfies unforgeability with the adversary $\mathcal{A}$ given access to the bidding oracle $\operatorname{Bid}\left(\mathrm{pk}, \mathrm{sk}_{s}, \mathrm{pk}_{v}, \cdot\right)$ for bid $x$ as well, but without the ability to obtain $\left(c\right.$, sig, $\left.\tau_{1}, \tau_{2}\right)$ as a response.

Signer Anonymity: It satisfies anonymity if for any PPT adversary $\mathcal{A}=\left(\mathcal{A}_{1}, \mathcal{A}_{2}\right)$,

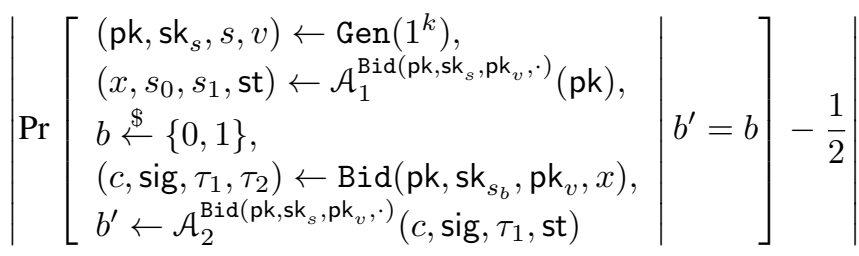

is negl $(k)$, where st is a state ensuring stateful communication between $\mathcal{A}_{1}$ and $\mathcal{A}_{2}$. If the attacker has an additional access to the list of secret keys, while preserving signer anonymity, then the scheme has signer anonymity against full key exposure.

Unpretendability: It satisfies unpretendability if for any PPT adversary $\mathcal{A}=\left(\mathcal{A}_{1}, \mathcal{A}_{2}\right)$,

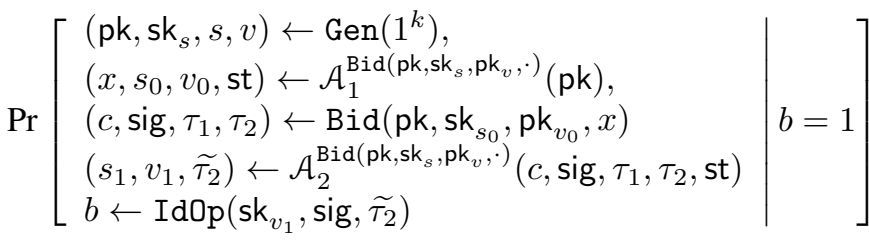

is negl $(k)$ when the adversary $\mathcal{A}$ is not allowed to choose $s_{0}=s_{1}$.

\section{Proposed Scheme}

Our proposed construction builds upon a DVRS introduced by Saraswat and Pandey in [9]. The resulting scheme provides correctness, unforgeability, unpretendability and bidder anonymity. In this context, the auctioneer is the designated verifier trusted to open the bids. However, a collusion between malicious bidders and the auctioneer does not affect the outcome.

There are three separate time frames for bid submission $\left[0, T_{1}\right]$, bid opening $\left[T_{1}, T_{2}\right]$ and winner declaration $\left[T_{2}, T_{3}\right]$. During the first time interval $\left[0, T_{1}\right]$, the bidders submit ring signed commitments of their bid and of their identity to the blockchain. During the second time interval $\left[T_{1}, T_{2}\right]$, the bidders reveal their bid by encrypting them using the public key of the auctioneer and sending them on chain. In the last interval $\left[T_{2}, T_{3}\right]$, the winning bidder reveals his identity to the auctioneer. In order to successfully realize our construction, we rely on the assumptions that the auctioneer is minimally trusted and assumed not to disclose the bidder's inputs; and that we assume the existence of a blockchain providing anonymity and confidentiality of the transactions on top of which our scheme can be implemented as a smart contract. This last assumption, despite the fact that it reduces the generality of the scheme, is not as strong as one might think. Indeed, the requested features can already be achieved, either by default in platforms such as Monero or Zcash [1], or in open blockchains such as Ethereum, by the addition of cryptographic protocols such as Zether [4], or stealth addressing. If our protocol is implemented on a platform with a built-in cryptocurrency, its security can be further improved by requiring the parties to deposit a given amount of cryptocurrency to the smart contract. The deposits get refunded to honest participants after the auction is concluded as an economic incentive.

\section{A. Our Generic Construction}

Our construction is based on four algorithms. The first one, represented in Algorithm 1, is the key generation algorithm, KeyGen, which generates the keys and the rings. It can be used by the bidders and the auctioneer. The same keys are used for signature and encryption.

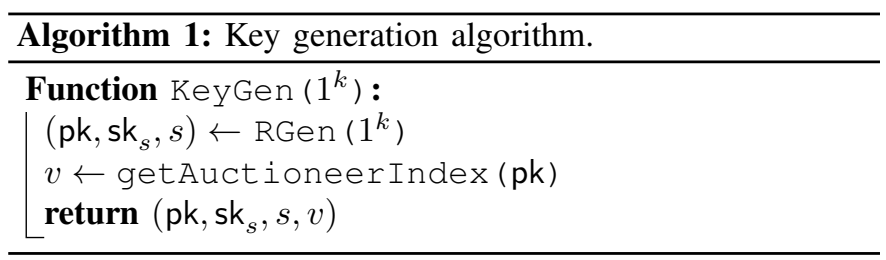

The second one, shown in Algorithm 2 is the bid submission algorithm. The bidders use it to compute the commitment $c$ to the bid value that they choose, as well as sig $=\sigma\left\|c_{1}\right\| c_{2}$, which is chain of commitments on encrypted ring signatures. This approach allows to link the values of the different tokens in such a way that they cannot be changed later during the auction protocol. This algorithm also outputs the bid opening token $\tau_{1}=C_{1} \| d_{1}$ and the identity opening token $\tau_{2}=C_{2} \| d_{2}$. Two different rings of parties are used in this algorithm. The first one is arbitrarily constructed by the bidder to maintain his anonymity. The second one is used to ensure that the auctioneer cannot convince anyone that the bidder actually generated a given bid, since she is also included in the ring. For the sake of generality, two different RS schemes can be used, RSig and R2Sig in this case.

Algorithm 3 shows the bid opening algorithm, BidOp, which is used to verify that the bid is valid, and to open and store its value. The bid opening token $\tau_{1}$ generated by the bidder is required. If the signatures and the commitments are successfully verified, the auctioneer stores locally the bid $x$ and the corresponding opening value $d$.

Finally, IdOp the identity opening algorithm shown in Algorithm 4 allows the auctioneer to check that the identity of the winning bidder is valid and to obtain his public key. The corresponding identity opening token $\tau_{2}$ is needed. The RS is verified using the ring made of the public keys of the winning bidder and the auctioneer only. If the identity is successfully 

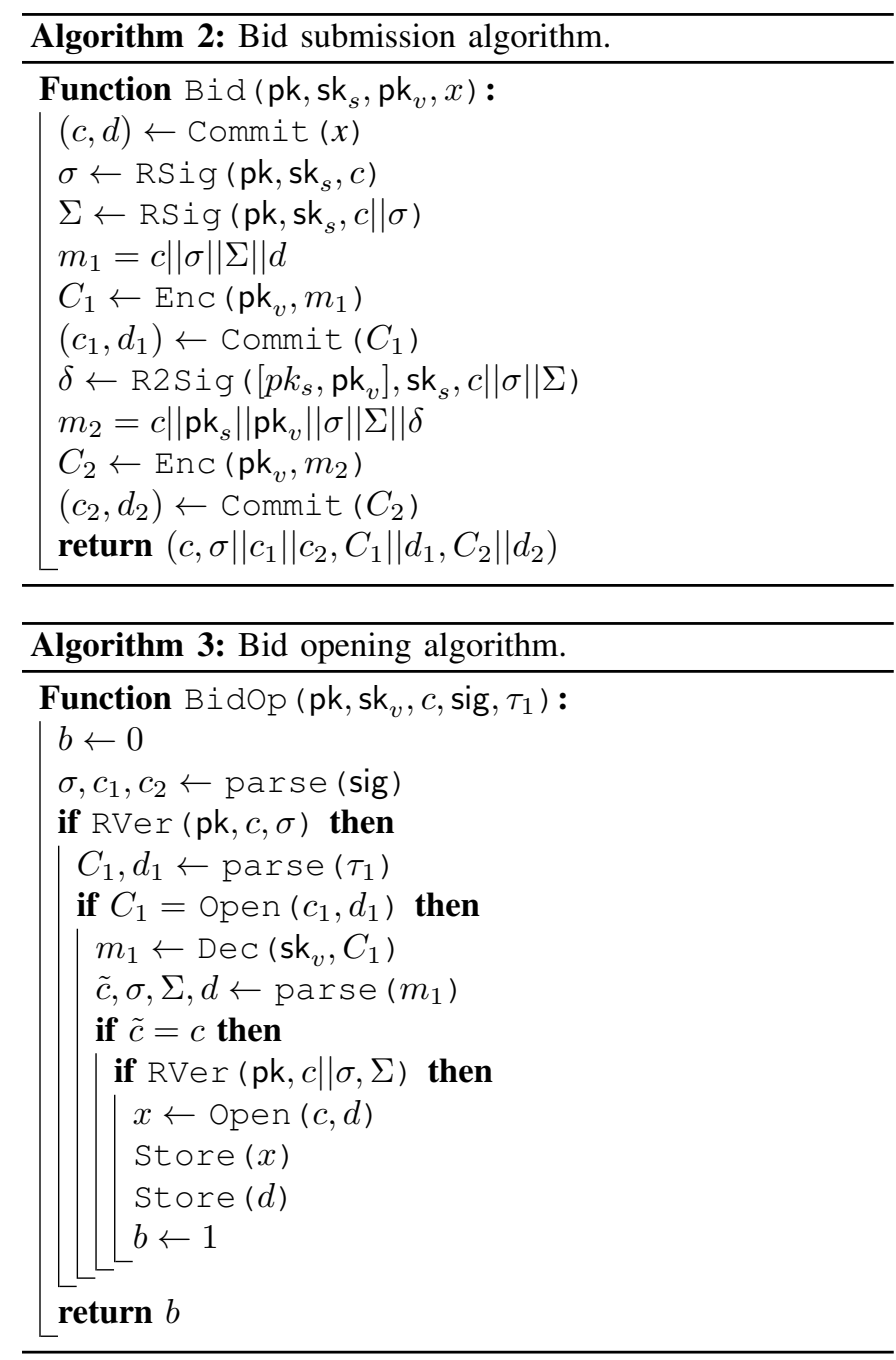

verified, the winning bidder's public key is stored locally by the auctioneer.

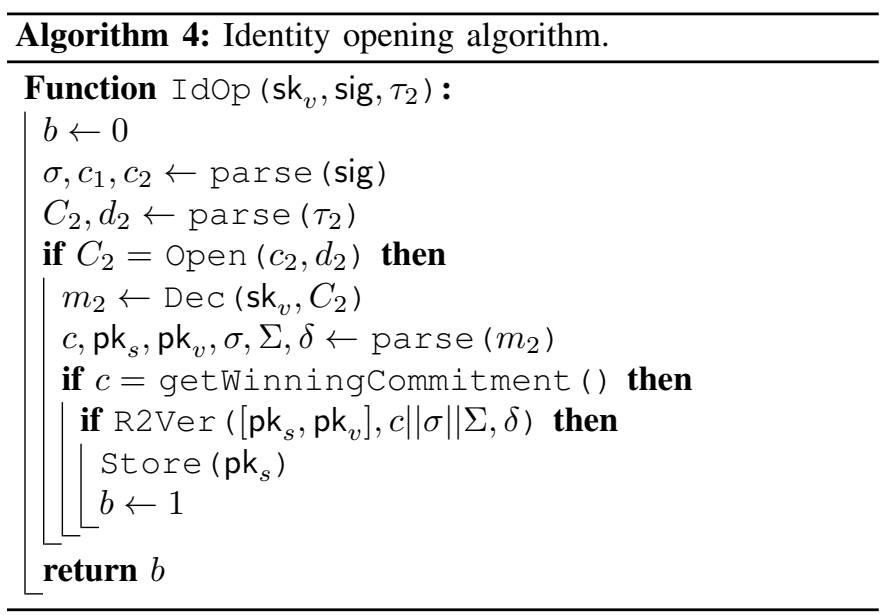

\section{B. Anonymous Fair Auction Protocol}

The protocol implementing our construction is presented in Algorithm 5. The first step is concerned with the generation of the keys for the auctioneer and the bidders. Before time $T_{1}$, each bidder chooses a bid and executes Bid, to get the 4-tuple $\left(c_{i}, \operatorname{sig}_{i}, \tau_{i 1}, \tau_{i 2}\right)$, sends $c_{i}$ and $\operatorname{sig}_{i}$ to the blockchain and stores locally the opening tokens.

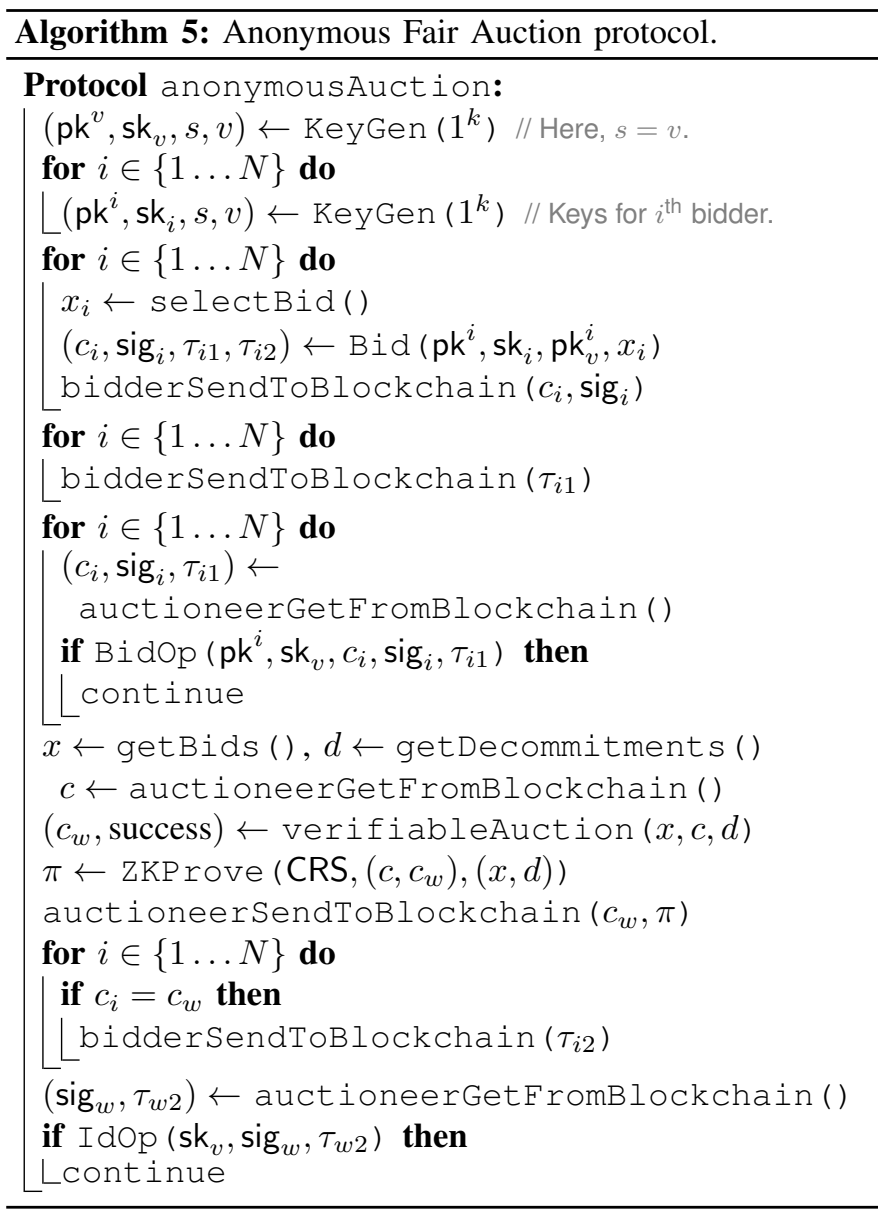

Between times $T_{1}$ and $T_{2}$, the bidders submit their respective bid opening token to the blockchain. The auctioneer fetches them, verifies the bids and stores their value. Then, the auctioneer computes the winning bid using the verifiableAuction algorithm and sends the commitment to the winning bid and the ZKP $\pi$ to the blockchain. Finally, between times $T_{2}$ and $T_{3}$, the winning bidder submits his identity opening token. The auctioneer verifies the identity and stores the winner's public key. During the whole auction process, the interactions between the auctioneer, the bidders and the smart contract can be listed as follows:

- $\mathrm{Tx}_{1}$ : The auctioneer triggers the auction smart contract.

- $\mathrm{Tx}_{2}: c$ and sig output by Bid are submitted by each bidder to the smart contract.

- $\mathrm{Tx}_{3}: \tau_{1}$ is submitted by each bidder to the smart contract.

- $\mathrm{Tx}_{4}$ : The auctioneer declares the commitment to the winning bid and submits proof $\pi$.

- $\mathrm{Tx}_{5}: \tau_{2}$ is submitted by the winning bidder.

- $\mathrm{Tx}_{6}$ : Auctioneer closes the auction.

\section{Zero-Knowledge Proof}

The ZKP system used in our scheme is shown in Algorithm 6 and is a slightly modified version of the one proposed in [5], where they designed their algorithm to handle a Vickrey 
auction, which implies that they have to return both the highest and the second highest bids. In our approach, we want to implement a first-price sealed-bid auction. Moreover, we do not want to disclose the value of the winning bid, hence the fact that our algorithm only returns its commitment. We also assume that all the bids are different. Regarding the generation of the CRS, if this is done in a trusted, centralized way by the auctioneer, then a malicious auctioneer could compute fake ZKPs. Therefore, to ensure that the system is secure against a malicious auctioneer, the CRS setup should be realised by all the parties involved and by using Multi-Party Computation, as it is suggested in [3].

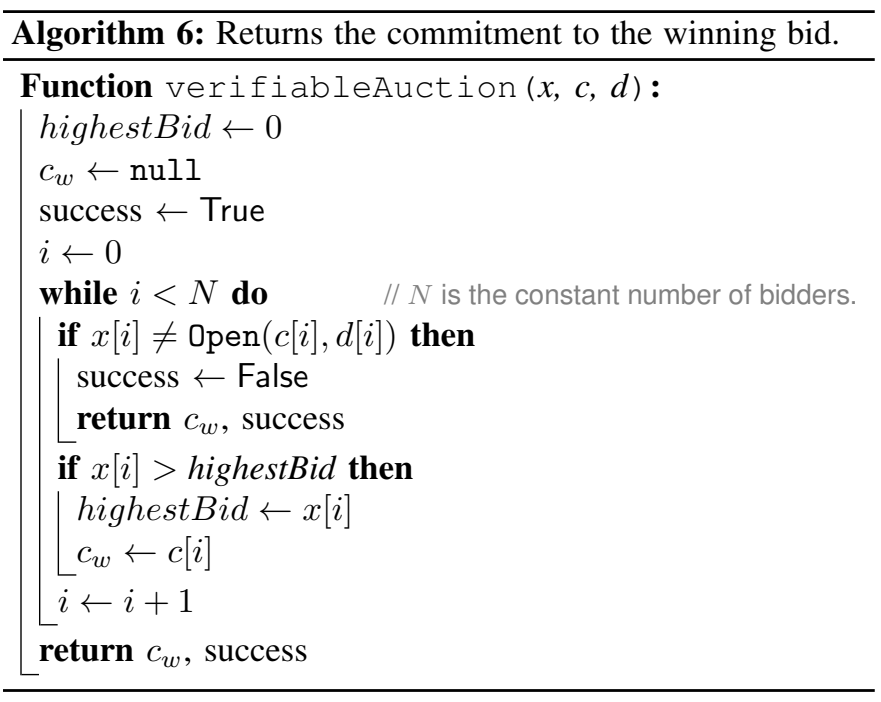

When the bidders have revealed their bids, the auctioneer executes Algorithm 6 and determines $c_{w}$, which is the commitment to the highest bid stored on the blockchain, and is able to compute the proof $\pi$. In this situation, the publicly known statement, is the $(N+1)$-tuple $\left(c ; c_{w}\right)$. The witness is the $2 N$-tuple $(x, d)$, which is a made of the value of the bids as well as their opening value. The smart contract verifies the auction thanks to the proof $\pi$ and to $\left(c ; c_{w}\right)$ by running $b \leftarrow$ ZKVer (CRS, $\left.\pi,\left(c ; c_{w}\right)\right)$. Since the CRS, $\pi$ and $\left(c ; c_{x}\right)$ are stored on the blockchain, one can leverage the auditability of inherent to this data structure to be convinced that the auction was executed correctly in a transparent, trustless configuration.

\section{SeCurity Proof}

In this section, we analyze the security of our proposed scheme.

Theorem 1. Assume RS = (RGen, RSig, RVer) be an anonymous and unforgeable ring signature, $\mathrm{PKE}=$ (PKEGen, Enc, Dec) be a semantically secure encryption scheme and Comm = (Commit,Open) be a hiding and binding commitment scheme, then our construction $\mathrm{ABS}=$ (KeyGen, Bid, BidOp, IdOp) relying on these components is correct, signer anonymous, unforgeable and unpretendable.

Proof. In order to prove this, we need to successfully deduce the security requirements of our construction from the corresponding security requirements of the underlying components.
Although the proof is straightforward, however, due to page restrictions, we defer it to the full version of this paper.

\section{CONCLUSION AND Future WORK}

We have introduced a generic protocol for anonymous fair auction using only standard cryptographic building blocks. The confidentiality and anonymity properties are achieved using DVRS and the transparency and auditability of blockchain platforms is leveraged in order to make the auction process publicly verifiable. In a future work, we intend to investigate the complexity and vulnerabilities that a blockchain environment might introduce.

\section{REFERENCES}

[1] E. Ben-Sasson, A. Chiesa, C. Garman, M. Green, I. Miers, E. Tromer, and M. Virza. Zerocash: decentralized anonymous payments from bitcoin. In 2014 IEEE Symposium on Security and Privacy, pages 459-474. IEEE, 2014. DOI: 10.1109/SP.2014.36. (Visited on 06/22/2020).

[2] E. Blass and F. Kerschbaum. Strain: A secure auction for blockchains. In ESORICS, volume 11098 of LNCS, pages 87-110. Springer, 2018.

[3] S. Bowe, A. Gabizon, and M. D. Green. A multi-party protocol for constructing the public parameters of the pinocchio zk-snark. In A. Zohar, I. Eyal, V. Teague, J. Clark, A. Bracciali, F. Pintore, and M. Sala, editors, Financial Cryptography and Data Security, pages 64-77, Berlin, Heidelberg. Springer Berlin Heidelberg, 2019. ISBN: 978-3-662-58820-8. DOI: 10 . 1007/978-3-662 58820 - 8_5. Cryptology ePrint Archive: $2017 / 602$. (Visited on 03/19/2021).

[4] B. Bünz, S. Agrawal, M. Zamani, and D. Boneh. Zether: towards privacy in a smart contract world. LNCS, 12059:423-443, 2020.

[5] H. S. Galal and A. M. Youssef. Succinctly verifiable sealed-bid auction smart contract. In Data Privacy Management, Cryptocurrencies and Blockchain Technology. Volume 11025, LNCS, pages 3-19. Springer, 2018.

[6] H. S. Galal and A. M. Youssef. Verifiable sealedbid auction on the ethereum blockchain. In Financial Cryptography, volume 10958 of LNCS, pages 265-278. Springer, 2018.

[7] A. E. Kosba, A. Miller, E. Shi, Z. Wen, and C. Papamanthou. Hawk: the blockchain model of cryptography and privacy-preserving smart contracts. In IEEE Symposium on Security and Privacy, pages 839-858. IEEE, 2016.

[8] R. L. Rivest, A. Shamir, and Y. Tauman. How to leak a secret. In ASIACRYPT, volume 2248 of LNCS, pages 552-565. Springer, 2001.

[9] V. Saraswat and S. K. Pandey. How to leak a secret and reap the rewards too. In LATINCRYPT, volume 8895 of LNCS, pages 348-367. Springer, 2014. 